\title{
Cardiac and gastric interoception have distinct neural substrates
}

Running title: Distinct substrates for cardiac/gastric interoception

$$
\text { Yusuke Haruki }{ }^{1} \text { and Kenji Ogawa }{ }^{1}
$$

${ }^{1}$ Department of Psychology, Graduate School of Humanities and Human Sciences, Hokkaido University, Kita 10, Nishi 7, Kita-ku, Sapporo 060-0810, Japan

Corresponding:

Yusuke Haruki

Department of Psychology, Graduate School of Humanities and Human Sciences, Hokkaido University, Kita 10, Nishi 7, Kita-ku, Sapporo 060-0810, Japan

E-mail: haruki@let.hokudai.ac.jp

Kenji Ogawa, Ph.D.

Department of Psychology, Faculty of Humanities and Human Sciences, Hokkaido University, Kita 10, Nishi 7, Kita-ku, Sapporo 060-0810, Japan

E-mail: ogawa@let.hokudai.ac.jp 


\begin{abstract}
The functional roles of interoception or senses that arise from within the body have been thought to guide the agents in adaptive behavior by informing of various bodily states such as a heart rate or energy status. However, direct evidence that the different types of interoception are processed or represented differently in the human brain is still lacking. Here, we directly compared the neural activation for cardiac (awareness related to heartbeats) and gastric (awareness related to stomach) interoception in the same population $(N=31)$ where the participants were asked to focus on sensations of their heart and stomach in the magnetic resonance imaging scanner. The result showed that neural activation underlying gastric interoception encompassed the sensorimotor and rewardrelated regions when compared to cardiac interoception, including the occipitotemporal visual cortices, bilateral primary motor cortex, primary somatosensory cortex, left orbitofrontal cortex, and bilateral hippocampal regions. Conversely, cardiac interoception selectively activated the right frontal operculum/insula in contrast to gastric interoception. Moreover, we found that the left dorsal middle insula encoded cardiac and gastric interoception differently but the posterior insula was not by performing multivoxel pattern analysis. Our results demonstrated that cardiac and gastric interoception, different types of bodily awareness, have distinct neural substrates particularly relevant to the functions for each interoception for the first time.
\end{abstract}




\section{Introduction}

The brain continuously receives numerous inputs from the peripheral nervous system. Recent studies have considered that such bodily or interoceptive signals would influence one's perceptual, affective and cognitive processes (Azzalini et al., 2019; Critchley and Garfinkel, 2018; Weng et al., 2021). Here, we specifically refer to awareness of signals that arise from within the body as interoception. Various types of interoception serve as an indicator of ongoing bodily states, guiding agents in adaptive behavior (Chen et al., 2021; Khalsa et al., 2018): thirst would drive an agent to drink water to keep hydrated, heartbeat perception inform of heightened arousal to be treated, and stomach emptiness tell when and where to eat. Nevertheless, it has not been fully elucidated how the human brain represents the various types of interoception. In the present study, we investigated whether neural substrates for cardiac interoception (awareness related to the heartbeat) and gastric interoception (awareness related to the stomach) differed.

Considering situations where people get aware of their heartbeats, the functional role of cardiac interoception would be to inform of changes in physiological arousal (elicited by such as fatigue or potential threats) and to guide appropriate action depending on the context; in contrast, gastric interoception is presumably related to foraging and feeding behavior by providing an ongoing nutritive status (Paulus et al., 2019; Quigley et al., 2021). Previous studies have found that accelerated false cardiac feedback (that is, exaggerated cardiac interoception) can modulate salience detection for an emotional picture (Gray et al., 2007) and perceived physical effort (Iodice et al., 2019). Also, an altered gastric interoception has been observed in people with an eating disorder compared with healthy controls (Van Dyck et al., 2021). Based on the functional difference in cardiac interoception and gastric interoception, we speculated that they would have distinct neural representation; specifically, cardiac interoception and gastric interoception may be encoded in the 
brain regions that subserve the functional roles of each interoception. In fact, visceral stimulations have been found to elicit a viscerotopical brain response in humans (Jarrahi et al., 2015; Lu et al., 2004). However, neuroimaging studies on interoception have extensively used cardiac interoception because of its easiness to perceive for participants (Critchley et al., 2004; Kuehn et al., 2015; Tan et al., 2018). Even when the brain activation of another modality of interoception (such as gastric or urinary) was measured, direct comparison of brain activation within the modality of interoception has not been discussed (Deville et al., 2020; Kerr et al., 2016; Simmons et al., 2013). Therefore, understanding of how the brain encodes and represents different types of interoception is still limited; for example, the right anterior insula has been suggested as the most relevant region for the subjective experience of cardiac interoception (Caseras et al., 2013; Pollatos et al., 2007) but some studies have questioned the generalizability of this relationship for another modality of interoception (Baltazar et al., 2021; Haruki and Ogawa, 2021).

To test whether cardiac interoception and gastric interoception are represented differently in the brain, we directly compared neural activation for cardiac interoception and gastric interoception in a healthy population using functional magnetic resonance imaging (fMRI). Specifically, we hypothesized that cardiac interoception would primarily activate the regions underlying the detection of physiological arousal, while gastric interoception would enhance activation in sensorimotor areas relevant to food intake. We also hypothesized that the insula, the most relevant architecture for interoception that receives an initial cortical input of interoceptive signals in the posterior region and represents a subjective experience of interoception in the middle-anterior region (Evrard, 2019; Oppenheimer and Cechetto, 2016), would exhibit a subregion-specific representation for cardiac and gastric interoception. Therefore, we performed ROI analysis in the subregions of the insula, combining multivoxel pattern analysis (MVPA) with a basic comparison of the neural activation. 


\section{Results}

\section{Overview}

We analyzed data obtained from 31 participants who participated in the fMRI experiment. In the MRI scanner, participants were asked to focus on sensations from their heart and stomach or visual stimuli (Figure 1). Runs were repeated for five times; at the end of each run, participants rated the subjective intensity of sensations through the run by using a Likert scale from 1 (no intense) to 7 (extremely).

\section{Subjective intensity of the sensations}

We performed a linear mixed effects model analysis (LME) for the subjective rating of the intensity of each sensation. The LME included the type of stimulus (heart, stomach, and visual) as the main factor and the effects of the participant and the sequence of runs as the random effects. Results indicated that subjective intensity of visual stimuli was rated the highest among the three (marginal mean contrasted to heart $=0.90, t(428.00)=7.59, p<.001)$ and the rating for stomach was the lowest (marginal mean $=-0.41, t(428.00)=-3.44, p<.001)$.

\section{Distinct brain activation for cardiac/gastric interoception}

By comparing whole-brain activation between cardiac and gastric interoception, we found that the sensorimotor and reward-related brain regions including the occipitotemporal visual cortices, bilateral primary motor, primary somatosensory, left orbitofrontal, and bilateral posterior hippocampus were activated in gastric interoception compared to cardiac interoception. In contrast, the right dorsal anterior insula extending to the frontal operculum only showed higher activation in cardiac than gastric interoception $(p<.001$, uncorrected; with cluster size correction for family wise 
error, corresponding to more than 41 voxels) (Figure 2A, Table 1). In particular, the visual area enhanced in gastric interoception was largely overlapped with previously reported brain regions that were coupled with the gastric basal rhythm (Rebollo et al., 2018, Figure 2B).

\section{Multivoxel pattern classification for cardiac/gastric interoception in the insula}

We trained a support vector machine (SVM) classifier for the brain activation pattern with respect to cardiac and gastric interoception. Activation patterns in anatomical subregions of the insular cortex (Faillenot et al., 2017, Figure 3A), the most relevant region in interoception, were used as inputs to the SVM. The classification accuracy in the left posterior short gyrus (PSG) which corresponds to the dorsal middle insula was significantly above the chance level (50\%) (mean classification accuracy $=56.32$, corrected $p$ value for false discovery rate $=.024$ ) (Figure 3B). A similar significance trend was also observed in the right anterior short gyrus (mean $=53.48$, corrected $p$ $=.089)$, left anterior short gyrus (mean $=54.26$, corrected $p=.089)$, and in the left middle short gyrus $($ mean $=53.42$, corrected $p=.089)$, but the other subdivisions of the insula showed no significant classification accuracy (corrected $p \mathrm{~s}>.27)$.

\section{Comparison of the signal change for cardiac/gastric interoception in the insula}

To directly compare the signal change in the insula subregions for cardiac and gastric interoception, parameter estimates for each condition were extracted from the insula ROIs (12 values for each condition). We then conducted a repeated-measures analysis of variance with the anatomical location and condition as the within-factors, independently for each hemisphere. The results indicated that in both hemispheres, the main effect of anatomical location and the interaction between location and condition were significant (left, $F(5,150)=56.37, \eta_{p}=.65 ; F(5,150)=8.89, \eta_{p}=.22$; right, $F(5$, $150)=39.18, F(5,150)=15.42, \eta_{p}=.34$; all $\left.p \mathrm{~s}<.001\right)$ but the main effect of condition was not (left, 
$F(1,30)=0.07, \eta_{p}=.00$; right, $\left.F(1,30)=0.15, \eta_{p}=.01 ; p \mathrm{~s}>.70\right)$ (Figure $3 \mathrm{C}$ ). Post-hoc analysis on the left ROIs showed a significance trend in the anterior long gyrus (ALG, corresponding to the dorsal posterior insula) where gastric interoception implied to show a higher activation compared with cardiac interoception $(p=.06)$. Post-hoc analysis also revealed that the right anterior short gyrus (ASG, dorsal anterior insula) and middle short gyrus (MSG, dorsal middle insula) exhibited a higher activation in cardiac than gastric interoception $(p=.02$, respectively). Multiple comparison for posthoc analysis was corrected by Shaffer's modified sequentially rejective Bonferroni procedure. 


\section{Discussion}

Our results demonstrate that cardiac and gastric interoception have distinct neural substrates. Specifically, the right anterior insula extending to frontal operculum that underlies detection of physiological salience showed higher activation in cardiac interoception. In contrast, gastric interoception enhanced the neural activation in the sensorimotor and reward-related brain regions, including the occipitotemporal visual cortices, bilateral primary motor, primary somatosensory, left orbitofrontal, and bilateral posterior hippocampus. These results suggest that a particular type of interoception is encoded in the brain region relevant to the functions of each interoception.

Previous studies have extensively studied heartbeat perception as a representative of interoception in general, suggesting the right anterior insula as the most relevant region for subjective experience of interoception (Craig, 2009; Critchley and Garfinkel, 2017). The present result, however, may question the general role of the right anterior insula by proposing that cardiac interoception would be processed dominantly in the right anterior insula than another modality (i.e., gastric). For example, individual accuracy of cardiac interoception has been consistently associated with activation in the right anterior insula (Caseras et al., 2013; Critchley et al., 2004; Pollatos et al., 2007), but it was not the case for awareness of breathing (Wang et al., 2019) or skin conductance (Baltazar et al., 2021). More important, increased cardiac interoception has been shown to modulate saliency detection: accelerated false cardiac feedback enhanced visual saliency for emotionally ambiguous stimuli, which was mediated by activation in the right anterior insula (Critchley and Garfinkel, 2015; Gray et al., 2007). These findings suggest that cardiac interoception would be processed dominantly in the right anterior insula to influence cognition and behavior by informing of a change in physiological arousal. This idea is in accordance with the recently proposed role of the right anterior insula that 
would encode the degree of subjective salience, whether it was influenced by interoceptive, emotional, or cognitive domain (Uddin, 2015). Taken together, our finding strongly suggests that the activation in the right anterior insula is strongly associated with cardiac interoception than other modalities, which may reflect the functional role of cardiac interoception that conveys the physiological arousal to be perceived.

We also found that the brain regions underlying gastric interoception largely encompassed the visual cortex compared with cardiac interoception. The involvement of the visual cortices in gastric interoception appears puzzling, but the converging evidence suggests the strong connection between the visual cortex and the stomach. Recently, Rebollo and colleagues have found the temporally delayed connectivity between the brain activity and the intrinsic electrical rhythm generated by the stomach, which encompassed the occipitotemporal visual cortices besides the somatosensory and motor area (Rebollo et al., 2018). The visual areas included in Rebollo and colleague's gastric network are largely overlapped with the cluster activated in the current experiment. Furthermore, electrical or vibration stimulus on the stomach has been confirmed to evoke neural activation in the visual area of the occipital area in rats (Cao et al., 2019), cats (Pigarev et al., 2013), and even in humans (Mayeli et al., 2021). Taken together, the gastric functions would be strongly tied with the visual cortex; here, we demonstrated that gastric interoception activated the visual cortex even without any explicit stimulation on the stomach.

The reason for the involvement of visual area may be that gastric functions are closely related to foraging and feeding behavior that require an integration of visuospatial information with the energy status and motor coordination (Kanoski and Grill, 2017; Rebollo et al., 2018). In line with this idea, we observed activations related to gastric interoception in the left orbitofrontal cortex, bilateral 
hippocampus, and primary motor cortex, as well as visual areas, which are all associated with food intake. For example, the orbitofrontal cortex has been suggested to modulate eating behavior by encoding the nutritive and reward values for food or food cues (Seabrook and Borgland, 2020); in fact, a meta-analysis for the fMRI studies of viewing food picture compared with non-food picture found the left orbitofrontal cortex as the most consistent activation for visual food stimuli (van der Laan et al., 2011). Furthermore, the role of the hippocampus in controlling food intake and regulating energy status has received much attention in the past few years (Quigley et al., 2021; Suarez et al., 2019). Recent evidence indicated that the human hippocampus encodes ongoing nutritive states in response to food cues (Jones et al., 2021). Based on these findings, we may consider that the neural responses to gastric interoception could be encoded in relation to food intake, which were activated by focusing on the bodily sensation without any stimulation or food-related cues in the present study.

The insular cortex has been regarded as the core region of interoception, with collecting, integrating, ang representing interoceptive signals along with the posterior-anterior gradient structure (Craig, 2009). Here, we identified the left posterior short gyrus (PSG, corresponding to the middle insula) in differently representing cardiac and gastric interoception by using MVPA. However, the dorsal posterior region of the insula, or the 'primary' interoceptive cortex that would receive an initial cortical input of interoceptive signals and have viscerotopic representation (Barrett and Simmons, 2015; Evrard, 2019), did not show the distinguishable activation pattern for cardiac and gastric interoception. On the contrary, we found no activation compared with baseline in the posterior insula. One possible explanation is that the primary interoceptive cortex would respond to changes in bodily states, rather than subjective experience of them: a recent study using rodents revealed that almost all neurons in the posterior insula responded to physiological changes (Cechetto and Saper, 1987; Gehrlach et al., 2019). In fact, interoceptive attention task where participants focus on their ongoing 
bodily sensations has been found to consistently activate the middle insula but the posterior (Haruki and Ogawa, 2021; Simmons et al., 2013). Rather, the posterior insula has been found to be functionally disconnected from other brain regions when people with accurate interoception performed the interoceptive attention task (Kuehn et al., 2015). Converging evidence suggests that the activation in the posterior insula is not indispensable for subjective experience of interoception. Our findings that the middle insula, rather than the posterior, showed the distinct activation pattern for cardiac and gastric interoception would contribute to elucidating the neural mechanism of how interoception to be perceived particularly in the insular cortex.

In summary, cardiac and gastric interoception were found to have distinct neural substrates: cardiac interoception was dominantly processed in the core of salience detection while the sensorimotor and reward-related regions were associated with gastric interoception. The middle insula, but not the posterior, encoded cardiac and gastric interoception differently. Future research should combine paradigms such as salience detection or presentation of food-related cues with the task of interoception to conclusively determine whether the neural encodings of interoception are related to its function as we suggested. Unfortunately, we did not employ any stimulus in the preset study; therefore, comparing interoception evoked by direct stimulation on the viscera and interoception at rest in the same population may provide more sophisticated insights regarding the functional mapping of interoeption. 


\section{Materials and methods}

\section{Participants}

A total of 35 people participated in the experiment (15 women). One of the participants (woman) requested an interruption during the experiment, yielding an incomplete data. Three of the rest of them (one man and two women) was excluded from the analysis due to their maximum head movement more than $3 \mathrm{~mm}$ during the experiment. Thus, the final analysis included thirty-one (12 women) participants who were $21.61 \pm 2.45$ years old (range: 20 to 31 ). Their handedness was assessed by a modified version of the Edinburgh Handedness Inventory for Japanese participants (Hatta and Nakatsuka, 1975). The sample size was determined to ensure reliable activations for interoception based on previous studies (Haruki and Ogawa, 2021; Wiebking and Northoff, 2015). Written informed consent was obtained from all participants. This study was carried out in accordance with the Declaration of Helsinki and all its future amendments. The Ethics Committee of Hokkaido University approved the experimental protocol.

\section{Task procedures}

To isolate the neural activation for cardiac and gastric interoception, we modified the task procedure used in previous studies (Deville et al., 2020; Kerr et al., 2016; Simmons et al., 2013). There were three types of task trial: heart (for cardiac interoception), stomach (gastric interoception), and visual trial (for control). Participants were asked to focus on sensations from their heart, stomach, or visual stimuli. In the heart and stomach trial, the words "HEART" and "STOMACH" were presented for each on the screen to let the participants find which task they are engaged in. In the visual trial, the word "TARGET" was presented on the screen, with the color of the word slowly and slightly faded from black to gray. The color changed every $1.5 \mathrm{~s}$ in total five times. By doing so, we tried to set the 
difficulty and situation of the trials as equal as possible; that is, in any task trial, participants focused on a particular vague sensation without any salient stimulus. In the rest period, participants were asked to watch a fixation crossbar and were prohibited preparing for the next task trial. Each trial lasted for $10 \mathrm{~s}$ and was followed by a $12 \mathrm{~s}$ lasting rest, which was presented five times for each trial: a total of 15 task trials was included per run. The order of task trials was pseudo-randomized, but the same trial was not presented three times in succession. At the end of each run, participants rated subjective intensity of the sensations (heart, stomach, and visual) during the run using a Likert scale from 1 (not intense at all) to 7 (extremely intense).

Approximately 5.5 min run was repeated five times for each participant to ensure a sufficient number of trials to perform MVPA. Before the first run began, all participants underwent a resting-state scanning that lasted for 5 min which was not analyzed in the present study. Stimuli were presented on a liquid crystal display and projected onto a custom-made viewing screen. The participants took a supine position in the scanner and viewed the screen via a mirror.

\section{MRI acquisition}

All scans were performed on a Siemens (Erlangen, Germany) 3-Tesla Prisma scanner with a 64channel head coil at Hokkaido University. T2*-weighted echo planar imaging (EPI) was used to acquire a total of 168 scans per run, with a gradient echo EPI sequence. The first three scans within each session were discarded in order to allow for T1 equilibration. The scanning parameters used were as follows: repetition time (TR), 2,000 ms; echo time (TE), $30 \mathrm{~ms}$; flip angle (FA), $90^{\circ}$; field of view (FOV), $192 \times 192$ mm; matrix, $94 \times 94$; 32 axial slices; and slice thickness, $3.500 \mathrm{~mm}$, with a $0.875 \mathrm{~mm}$ gap. Thus, the voxel size was $2.042 \times 2.042 \times 4.375 \mathrm{~mm}$. T1-weighted anatomical 
imaging with an MP-RAGE sequence was performed using the following parameters: TR, 2,300 ms; TE, $2.32 \mathrm{~ms}$; FA, $8^{\circ}$; FOV, $256 \times 256 \mathrm{~mm}$; matrix, $256 \times 256$; 192 axial slices; and slice thickness, 1 mm without a gap.

\section{Preprocessing of fMRI data}

Image preprocessing was performed using the SPM12 software (Wellcome Department of Cognitive Neurology, http://www.fil.ion.ucl.ac.uk/spm). All functional images were initially realigned to adjust for motion-related artifacts. Volume-based realignment was performed by co-registering images using rigid-body transformation to minimize the squared differences between volumes. The realigned images were then spatially normalized with the Montreal Neurological Institute (MNI) template based on the affine and non-linear registration of co-registered T1-weighted anatomical images. They were resampled into 3-mm-cube voxels with sinc interpolation. Images were spatially smoothed using a Gaussian kernel of $6 \times 6 \times 6-\mathrm{mm}$ full width at half-maximum. The images used for MVPA were not smoothed to avoid blurring the information contained in the multi-voxel activity pattern.

\section{Statistical analysis}

\section{Subjective intensity of the sensations}

We performed a linear mixed effects model analysis (LME) implemented in the lme4 package (Bates et al., 2015) in $\mathrm{R}$ (version 4.0.3) to test whether the subjective intensity of sensations differed between modalities (cardiac, gastric, and visual). With all the data obtained $(\mathrm{N}=465,3$ trial types for 5 runs for 31 participants) as a dependent variable, we modeled the type of sensation as the fixed effect and the effects of participants and runs as the random effects. The LMEs enabled us to avoid averaging the values across five runs compared to the traditional analysis of variance. The parameter 
was estimated using the restricted maximum likelihood method, with the degrees of freedom estimated using the Satterthwaite method.

\section{Whole-brain mass univariate analysis}

Individual level generalized linear model (GLM) included the 15 task trial blocks per run as separate box-car regressors that were convolved with the canonical hemodynamic response function. To reduce motion-related artifacts, six motion parameters were included as nuisance regressors. As a group level analysis, we first conducted whole-brain mass univariate analysis to directly compare the brain activation between cardiac and gastric interoception. Contrast images of the heart trial compared with the stomach trial and the opposite were created for each participant. We then performed a group level random effects analysis for these images using one sample t-test. The voxellevel threshold was set to a $p<.001$ (uncorrected) and the cluster size was corrected for family wise error (FWE) $(p<.05$, corresponds to more than 40 voxels).

\section{ROI analysis in subregions of the insula for cardiac and gastric interoception}

We explored distinct neural representation for cardiac and gastric interoception in subregions of the insula by using ROI-based multivoxel pattern analysis (MVPA) and by directly comparing the signal strength. We adopted the anatomical ROIs from Hammersmith brain atlases (Brain Development, www.brain-development.org). These images were constructed as a 3D probabilistic atlas using in vivo T1 MR images, including anatomical structures commonly seen in the human insula: the anterior short gyrus (ASG; the most dorsal anterior portion of the insula), middle short gyrus (MSG; the dorsal mid-anterior), posterior short gyrus (PSG; the dorsal mid-posterior), anterior inferior 
cortex (AIC; the ventral anterior), anterior long gyrus (ALG; the dorsal posterior), and posterior long gyrus (PLG; the ventral posterior) (Faillenot et al., 2017).

MVPA for cardiac and gastric interoception was performed with a two-class classifier based on a linear support vector machine (SVM) implemented in LIBSVM (http://www.csie.ntu.edu.tw/ cjlin/libsvm/). Parameter estimates for each trial of voxels within the ROIs were used as input to the classifier. Individual level classification accuracy was estimated with a five-fold "leave-one-out" cross-validation, where four runs were used as training, and one remaining run was used as test data. We used a default hyperparameter (a fixed regularization parameter $\mathrm{C}=1$ ). A one-sample t-test was performed to test whether each subregion of the insula exhibits statistically significant classification accuracy higher than chance $(50 \%)$ at group level. Because we used 12 ROIs (6 anatomical regions for both hemispheres), the calculated $p$ values were corrected for Benjamini and Hockberg's false discovery rate (FDR; Benjamini \& Hochberg, 1995).

To compare the signal strength in the insula subregions between cardiac and gastric interoception, we extracted the individual signal strength as beta weights for each condition. Then, we performed an analysis of variance on these values with the condition and anatomical location as the within-factors, independently for each hemisphere. 
bioRxiv preprint doi: https://doi.org/10.1101/2022.02.18.480981; this version posted February 20, 2022. The copyright holder for this preprint (which was not certified by peer review) is the author/funder, who has granted bioRxiv a license to display the preprint in perpetuity. It is made available under aCC-BY 4.0 International license.

\section{Acknowledgement}

This work was supported by JSPS KAKENHI Grant Number 20K20423. 


\section{Reference}

Azzalini D, Rebollo I, Tallon-Baudry C. 2019. Visceral Signals Shape Brain Dynamics and

Cognition. Trends Cogn Sci 23:488-509. doi:10.1016/j.tics.2019.03.007

Baltazar M, Picq J, Conty L. 2021. Neural correlates of interoceptive accuracy : Beyond cardioception. Eur J Neurosci 54:7642-7653. doi:10.1111/ejn.15510

Barrett LF, Simmons WK. 2015. Interoceptive predictions in the brain. Nat Rev Neurosci 16:419429. doi:10.1038/nrn3950.

Bates D, Mächler M, Bolker BM, Walker SC. 2015. Fitting linear mixed-effects models using lme4. J Stat Softw 67. doi:10.18637/jss.v067.i01

Benjamini Y, Hochberg Y. 1995. Controlling the False Discovery Rate: A Practical and Powerful Approach to Multiple Testing. J R Stat Soc Ser B 57:289-300.

Cao J, Lu KH, Oleson ST, Phillips RJ, Jaffey D, Hendren CL, Powley TL, Liu Z. 2019. Gastric stimulation drives fast BOLD responses of neural origin. Neuroimage 197:200-211. doi:10.1016/j.neuroimage.2019.04.064

Caseras X, Murphy K, Mataix-Cols D, López-Solà M, Soriano-Mas C, Ortriz H, Pujol J, Torrubia R. 2013. Anatomical and functional overlap within the insula and anterior cingulate cortex during interoception and phobic symptom provocation. Hum Brain Mapp 34:1220-1229. doi:10.1002/hbm.21503 
bioRxiv preprint doi: https://doi org/10.1101/2022.02 18.480981; this version posted February 20,2022 . The copyright holder for this preprint (which was not certified by peer review) is the author/funder, who has granted bioRxiv a license to display the preprint in perpetuity. It is made available under aCC-BY 4.0 International license.

Cechetto DF, Saper CB. 1987. Evidence for a viscerotopic sensory representation in the cortex and thalamus in the rat. J Comp Neurol 262:27-45. doi:10.1002/cne.902620104

Chen WG, Schloesser D, Arensdorf AM, Simmons JM, Cui C, Valentino R, Gnadt JW, Nielsen L, Hillaire-clarke CS, Spruance V, Horowitz TS, Vallejo YF, Langevin HM. 2021. The Emerging Science of Interoception : Sensing, Integrating, Interpreting, and Regulating Signals within the Self. Trends Neurosci 44:3-16. doi:10.1016/j.tins.2020.10.007

Craig AD. 2009. How do you feel — now? The anterior insula and human awareness. Nat Rev Neurosci 10:59-70. doi:10.1038/nrn2555

Critchley HD, Garfinkel SN. 2018. The influence of physiological signals on cognition. Curr Opin Behav Sci 19:13-18. doi:10.1016/j.cobeha.2017.08.014

Critchley HD, Garfinkel SN. 2017. Interoception and emotion. Curr Opin Psychol 17:7-14. doi:10.1016/j.copsyc.2017.04.020

Critchley HD, Garfinkel SN. 2015. Interactions between visceral afferent signaling and stimulus processing. Front Neurosci 9:00286. doi:10.3389/fnins.2015.00286

Critchley HD, Wiens S, Rotshtein P, Öhman A, Dolan RJ. 2004. Neural systems supporting interoceptive awareness. Nat Neurosci 7:189-195. doi:10.1038/nn1176 
Deville DC, Kuplicki R, Stewart JL, Paulus MP, Khalsa SS, Aupperle RL, Bodurka J, Cha YH, Feinstein J, Savitz JB, Victor TA. 2020. Diminished responses to bodily threat and blunted interoception in suicide attempters. Elife 9:e51593. doi:10.7554/eLife.51593

Evrard HC. 2019. The organization of the primate insular cortex. Front Neuroanat 13:00043.

doi:10.3389/fnana.2019.00043

Faillenot I, Heckemann RA, Frot M, Hammers A. 2017. Macroanatomy and 3D probabilistic atlas of the human insula. Neuroimage 150:88-98. doi:10.1016/j.neuroimage.2017.01.073

Gehrlach DA, Dolensek N, Klein AS, Roy Chowdhury R, Matthys A, Junghänel M, Gaitanos TN, Podgornik A, Black TD, Reddy Vaka N, Conzelmann KK, Gogolla N. 2019. Aversive state processing in the posterior insular cortex. Nat Neurosci 22:1424-1437. doi:10.1038/s41593019-0469-1

Gray MA, Harrison NA, Wiens S, Critchley HD. 2007. Modulation of emotional appraisal by false physiological feedback during fMRI. PLoS One 2. doi:10.1371/journal.pone.0000546

Haruki Y, Ogawa K. 2021. Role of anatomical insular subdivisions in interoception: Interoceptive attention and accuracy have dissociable substrates. Eur J Neurosci 53:2669-2680.

doi:10.1111/ejn.15157

Hatta T, Nakatsuka Z. 1975. Handedness inventoryPapers on Celebrating 63rd Birthday of Prof.

Ohnishi. Osaka City University. pp. 224-245. 
Iodice P, Porciello G, Bufalari I, Barca L, Pezzulo G. 2019. An interoceptive illusion of effort induced by false heart-rate feedback. Proc Natl Acad Sci U S A 116:13897-13902.

doi:10.1073/pnas.1821032116

Jarrahi B, Mantini D, Balsters JH, Michels L, Kessler TM, Mehnert U, Kollias SS. 2015. Differential functional brain network connectivity during visceral interoception as revealed by independent component analysis of fMRI time-series. Hum Brain Mapp 36:4438-4468.

doi:10.1002/hbm.22929

Jones S, Luo S, Dorton HM, Angelo B, Yunker AG, Monterosso JR, Page KA. 2021. Evidence of a Role for the Hippocampus in Food-Cue Processing and the Association with Body Weight and Dietary Added Sugar. Obesity 29:370-378. doi:10.1002/oby.23085

Kanoski SE, Grill HJ. 2017. Hippocampus Contributions to Food Intake Control: Mnemonic, Neuroanatomical, and Endocrine Mechanisms. Biol Psychiatry 81:748-756. doi:10.1016/j.biopsych.2015.09.011

Kerr KL, Moseman SE, Avery JA, Bodurka J, Zucker NL, Simmons WK. 2016. Altered insula activity during visceral interoception in weight-restored patients with anorexia nervosa. Neuropsychopharmacology 41:521-528. doi:10.1038/npp.2015.174

Khalsa SS, Adolphs R, Cameron OG, Critchley HD, Davenport PW, Feinstein JS, Feusner JD, Garfinkel SN, Lane RD, Mehling WE, Meuret AE, Nemeroff CB, Oppenheimer S, Petzschner 
FH, Pollatos O, Rhudy JL, Schramm LP, Simmons WK, Stein MB, Stephan KE, Van den Bergh

O, Van Diest I, von Leupoldt A, Paulus MP, Ainley V, Al Zoubi O, Aupperle R, Avery JA,

Baxter L, Benke C, Berner L, Bodurka J, Breese E, Brown T, Burrows K, Cha YH, Clausen A,

Cosgrove K, Deville D, Duncan L, Duquette P, Ekhtiari H, Fine T, Ford B, Garcia Cordero I,

Gleghorn D, Guereca Y, Harrison NA, Hassanpour M, Hechler T, Heller A, Hellman N, Herbert

B, Jarrahi B, Kerr K, Kirlic N, Klabunde M, Kraynak T, Kriegsman M, Kroll J, Kuplicki R,

Lapidus R, Le T, Hagen KL, Mayeli A, Morris A, Naqvi N, Oldroyd K, Pané-Farré C, Phillips

R, Poppa T, Potter W, Puhl M, Safron A, Sala M, Savitz J, Saxon H, Schoenhals W, Stanwell-

Smith C, Teed A, Terasawa Y, Thompson K, Toups M, Umeda S, Upshaw V, Victor T,

Wierenga C, Wohlrab C, Yeh H wen, Yoris A, Zeidan F, Zotev V, Zucker N. 2018.

Interoception and Mental Health: A Roadmap. Biol Psychiatry Cogn Neurosci Neuroimaging

3:501-513. doi:10.1016/j.bpsc.2017.12.004

Kuehn E, Mueller K, Lohmann G, Schuetz-Bosbach S. 2015. Interoceptive awareness changes the posterior insula functional connectivity profile. Brain Struct Funct 221:1555-1571.

doi:10.1007/s00429-015-0989-8

Lu CL, Wu YT, Yeh TC, Chen LF, Chang FY, Lee SD, Ho LT, Hsieh JC. 2004. Neuronal correlates of gastric pain induced by fundus distension: A 3T-fMRI study. Neurogastroenterol Motil 16:575-587. doi:10.1111/j.1365-2982.2004.00562.x 
bioRxiv preprint doi: https://doi org/10.1101/2022.02 18.480981· this version posted February 20,2022 . The copyright holder for this preprint (which was not certified by peer review) is the author/funder, who has granted bioRxiv a license to display the preprint in perpetuity. It is made available under aCC-BY 4.0 International license.

Mayeli A, Al Zoubi O, White EJ, Chappelle S, Kuplicki R, Smith R, Feinstein JS, Bodurka J, Paulus MP, Khalsa SS. 2021. Neural indicators of human gut feelings. bioRxiv.

doi:10.1101/2021.02.11.430867

Oppenheimer S, Cechetto D. 2016. The insular cortex and the regulation of cardiac function. Compr Physiol 6:1081-1133. doi:10.1002/cphy.c140076

Paulus MP, Feinstein JS, Khalsa SS. 2019. An Active Inference Approach to Interoceptive Psychopathology. Annu Rev Clin Psychol 15:97-122. doi:10.1146/annurev-clinpsy-050718095617

Pigarev IN, Bagaev VA, Levichkina E V, Fedorov GO, Busigina II. 2013. Cortical visual areas process intestinal information during slow-wave sleep. Neurogastroenterol Motil 25:268-e169. doi:10.1111/nmo.12052

Pollatos O, Schandry R, Auer DP, Kaufmann C. 2007. Brain structures mediating cardiovascular arousal and interoceptive awareness. Brain Res 1141:178-187.

doi:10.1016/j.brainres.2007.01.026

Quigley KS, Kanoski S, Grill WM, Barrett LF, Tsakiris M. 2021. Functions of Interoception: From Energy Regulation to Experience of the Self. Trends Neurosci 44:29-38.

doi:10.1016/j.tins.2020.09.008 
Rebollo I, Devauchelle AD, Béranger B, Tallon-Baudry C. 2018. Stomach-brain synchrony reveals a novel, delayed-connectivity resting-state network in humans. Elife 7:e33321.

doi:10.7554/eLife.33321

Seabrook LT, Borgland SL. 2020. The orbitofrontal cortex, food intake and obesity. J Psychiatry

Neurosci 45:304-312. doi:10.1503/jpn.190163

Simmons WK, Avery JA, Barcalow JC, Bodurka J, Drevets WC, Bellgowan P. 2013. Keeping the body in mind: Insula functional organization and functional connectivity integrate interoceptive, exteroceptive, and emotional awareness. Hum Brain Mapp 34:2944-2958.

doi:10.1002/hbm.22113

Suarez AN, Noble EE, Kanoski SE. 2019. Regulation of memory function by feeding-relevant biological systems: Following the breadcrumbs to the hippocampus. Front Mol Neurosci 12:00101. doi:10.3389/fnmol.2019.00101

Tan Y, Wei D, Zhang M, Yang J, Jelinčić V, Qiu J. 2018. The role of mid-insula in the relationship between cardiac interoceptive attention and anxiety: evidence from an fMRI study. Sci Rep 8:17280. doi:10.1038/s41598-018-35635-6

Uddin LQ. 2015. Salience processing and insular cortical function and dysfunction. Nat Rev Neurosci 16:55-61. doi:10.1038/nrn3857 
bioRxiv preprint doi: https://doi org/10.1101/2022.02 18.480981; this version posted February 20, 2022. The copyright holder for this preprint (which was not certified by peer review) is the author/funder, who has granted bioRxiv a license to display the preprint in perpetuity. It is made available under aCC-BY 4.0 International license.

van der Laan LN, de Ridder DTD, Viergever MA, Smeets PAM. 2011. The first taste is always with the eyes: A meta-analysis on the neural correlates of processing visual food cues. Neuroimage 55:296-303. doi:10.1016/j.neuroimage.2010.11.055

Van Dyck Z, Schulz A, Blechert J, Herbert BM, Lutz APCC, Vögele C, Dyck Z Van, Schulz A, Lutz APCC, Vögele C, Blechert J, Herbert BM. 2021. Gastric interoception and gastric myoelectrical activity in bulimia nervosa and binge-eating disorder. Int J Eat Disord 54:1106-1115. doi:10.1002/eat.23291

Wang X, Wu Q, Egan L, Gu X, Liu P, Gu H, Yang Y, Luo J, Wu Y, Gao Z, Fan J. 2019. Anterior insular cortex plays a critical role in interoceptive attention. Elife 8:e42265. doi:10.1101/464867

Weng HY, Feldman JL, Leggio L, Napadow V, Park J, Price CJ. 2021. Interventions and Manipulations of Interoception. Trends Neurosci 44:52-62. doi:10.1016/j.tins.2020.09.010

Wiebking C, Northoff G. 2015. Neural activity during interoceptive awareness and its associations with alexithymia-An fMRI study in major depressive disorder and non-psychiatric controls. Front Psychol 6:1-16. doi:10.3389/fpsyg.2015.00589 


\section{Figure legends}

\section{Figure 1. Schematical presentation of the task procedure.}

Example of time course of the functional magnetic resonance imaging (fMRI) task are depicted. Our block design fMRI task included three types of task trial (cardiac interoception, gastric interoception, and visual) that lasted $10 \mathrm{~s}$, which were followed by rest (12 s). Participants were asked to focus on sensations according to the type of task. At the end of each run, participants rated the subjective intensity of the sensations for each condition using a Likert scale from 1 (not intense at all) to 7 (extremely intense).

\section{Figure 2. Whole brain activation for cardiac and gastric interoception.}

A). Significant activation in cardiac interoception contrasted gastric interoception (warm color) and the opposite (cold color) are depicted with a height threshold $p<.001$ (uncorrected) and an extent threshold $p<.05$ (corrected for family wise error). Sagittal views show that cardiac and gastric interoception activated the brain region relevant to each interoception. Sensorimotor regions (S1: primary sensory, M1: primary motor, V3: visual area 3, FG: fusiform gyrus) and reward-related regions (OFC: orbitofrontal cortex, HP: hippocampus) were enhanced in gastric interoception, while the right anterior insula (AI) was activated in cardiac interoception. B). The internally extended visual area activated in gastric interoception was largely overlapped with the brain regions that have been shown to couple with the gastric basal rhythm (Rebollo et al., 2018). L, left hemisphere; R, right hemisphere. All figures are shown in axial, sagittal, and coronal slices with $\mathrm{z}, \mathrm{x}$, and y denoting respectively locations in the Montreal Neurological Institute (MNI) coordinates

Figure 3. Neural activation for cardiac and gastric interoception in the subdivisions of insula. 
A). The anatomical subdivisions of the insular cortex (Faillenot et al., 2017) are presented. We adopted six regions (the anterior short gyrus: ASG, middle short gyrus: MSG, posterior short gyrus: PSG, anterior insular cortex: AIC, anterior long gyrus: ALG, and posterior long gyrus: PLG) for both hemispheres. B). The results of multivoxel pattern analysis are depicted, with the left panel showing the results of left insula subdivision and the right panel showing the right insula. The left MSG exhibited significantly higher classification accuracy above chance level (50\%). A similar significance trend (that is, $p<.1$ ) was observed in the right ASG, left ASG and left MSG. All $p$ values were corrected for Benjamini and Hochberg's false discovery rate. The point plot represents the mean classification accuracy with $95 \%$ confidential interval while the half-violin plot represents the kernel density estimation. C). The results of direct comparison of the signal strength between cardiac and gastric interoception are plotted. Post-hoc analysis of repeated-measures analysis of variance revealed that the right ASG and MSG showed significantly higher activation in cardiac interoception than gastric interoception. There was a significance trend that implies stronger activation in gastric than cardiac interoception in the left ALG. Shaffer's modified sequentially rejective Bonferroni procedure was used for multiple comparison correction. The point plots represent the mean signal change with $95 \%$ confidential interval while the half-violin plot represents the kernel density estimation (green for cardiac, orange for gastric interoception). ${ }^{*}, p<.05 ; \dagger, p$ $<.10$ (corrected for false discovery rate) 
Table

Location

Voxels

MNI coordinates

$\mathrm{T}$ value

y

Contrast: cardiac interoception > gastric interoception

\begin{tabular}{llllll}
\hline R Dorsal anterior insula & 48 & 33 & 17 & 2 & 4.65 \\
Frontal operculum & & 54 & 11 & 8 & 4.39 \\
Dorsal anterior insula & & 30 & 26 & 2 & 3.72
\end{tabular}

Contrast: gastric interoception > cardiac interoception

\begin{tabular}{|c|c|c|c|c|c|}
\hline L Secondary visual area & 3793 & -12 & -73 & -13 & 8.42 \\
\hline $\mathrm{R}$ Primary visual area & & 9 & -82 & 5 & 8.29 \\
\hline Secondary visual area & & 18 & -79 & 5 & 8.06 \\
\hline L Middle temporal gyrus & 211 & -60 & -31 & -4 & 6.71 \\
\hline Superior temporal gyrus & & -57 & -7 & -7 & 4.64 \\
\hline Middle temporal gyrus & & -54 & -19 & -7 & 4.29 \\
\hline \multirow[t]{3}{*}{ L Lateral orbitofrontal cortex } & 95 & -42 & 26 & -16 & 6.21 \\
\hline & & -36 & 38 & -16 & 5.29 \\
\hline & & -51 & 26 & -4 & 3.99 \\
\hline L Primary motor cortex & 67 & -48 & -7 & 29 & 5.90 \\
\hline $\mathrm{R}$ Primary motor cortex & 41 & 48 & -7 & 35 & 5.88 \\
\hline \multirow[t]{3}{*}{ L Primary sensory area } & 116 & -12 & -25 & 59 & 5.50 \\
\hline & & -3 & -37 & 68 & 4.57 \\
\hline & & -18 & -37 & 68 & 4.26 \\
\hline \multirow[t]{3}{*}{ L Fusiform gyrus } & 181 & -51 & -58 & -13 & 5.31 \\
\hline & & -48 & -46 & -13 & 4.52 \\
\hline & & -36 & -61 & -13 & 4.18 \\
\hline \multirow[t]{2}{*}{ L Primary motor cortex } & 47 & -36 & -25 & 53 & 5.18 \\
\hline & & -39 & -22 & 65 & 3.71 \\
\hline \multirow[t]{2}{*}{ L Dorsomedial prefrontal cortex } & 96 & -12 & 62 & 17 & 5.14 \\
\hline & & -18 & 59 & 29 & 5.00 \\
\hline
\end{tabular}


continued

\begin{tabular}{|c|c|c|c|c|c|}
\hline & & -9 & 59 & 5 & 4.93 \\
\hline \multirow[t]{2}{*}{ L Medial orbitofrontal cortex } & 52 & -3 & 47 & -13 & 5.11 \\
\hline & & -3 & 56 & -10 & 4. \\
\hline \multirow[t]{2}{*}{ L Frontal eye field } & 50 & -21 & 32 & 53 & \\
\hline & & -12 & 35 & 44 & \\
\hline \multirow[t]{3}{*}{$\mathrm{R}$ Premotor area } & 50 & 33 & -22 & 53 & \\
\hline & & 33 & -10 & 68 & \\
\hline & & 33 & -25 & 62 & \\
\hline \multirow[t]{3}{*}{ L Dorsolateral prefrontal cortex } & 42 & -54 & 26 & 23 & 4. \\
\hline & & -39 & 26 & 14 & \\
\hline & & -48 & 29 & 5 & \\
\hline
\end{tabular}

Table 1. Anatomical regions, peak voxel coordinates, and t-values of observed activations.

The peak-level threshold was set to $p<.001$ and the cluster size was also corrected for extent threshold with family wise error ( $p<.05$, corresponding more than 42 voxels). L, left hemisphere; MNI, Montreal Neurological Institute; R, right hemisphere 
bioRxiv preprint doi: https://doi.org/10.1101/2022.02.18.480981; this version posted February 20, 2022. The copyright holder for this preprint (which was not certified by peer review) is the author/funder, who has granted bioRxiv a license to display the preprint in perpetuity. It is made available under aCC-BY 4.0 International license.

\section{Figures}

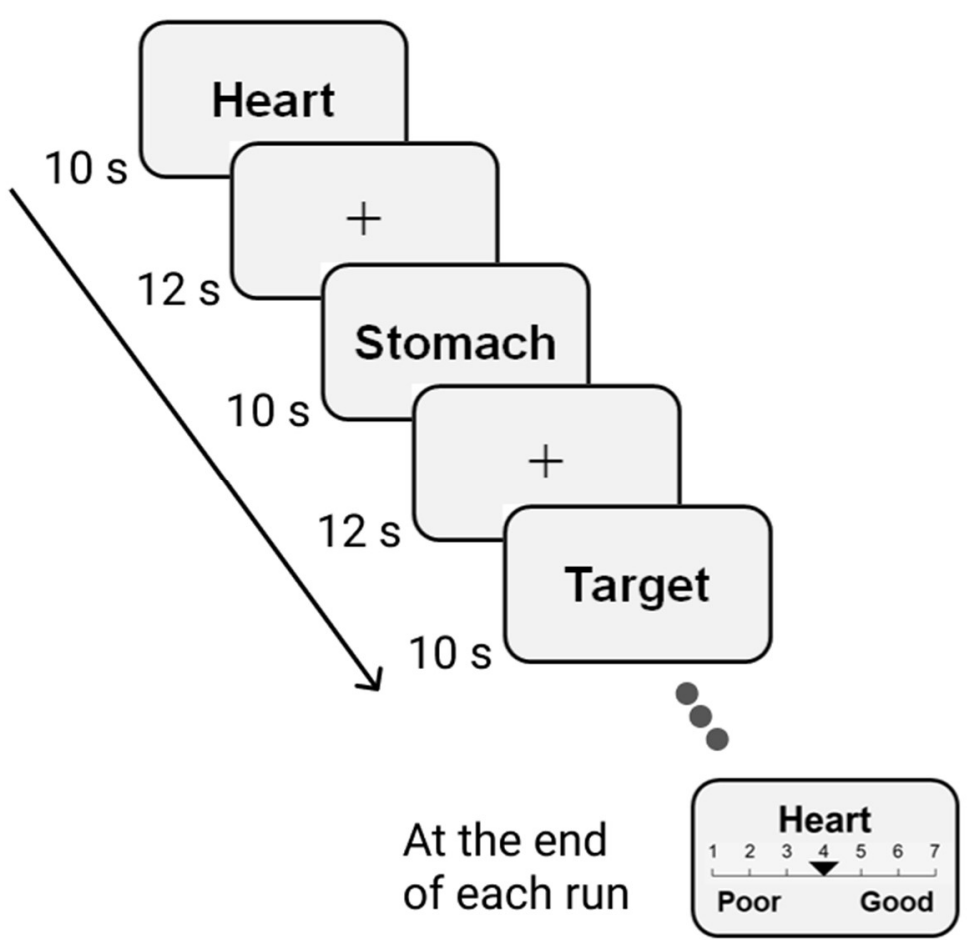


A

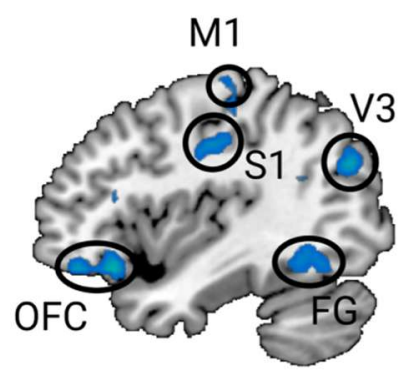

$$
x=-40
$$
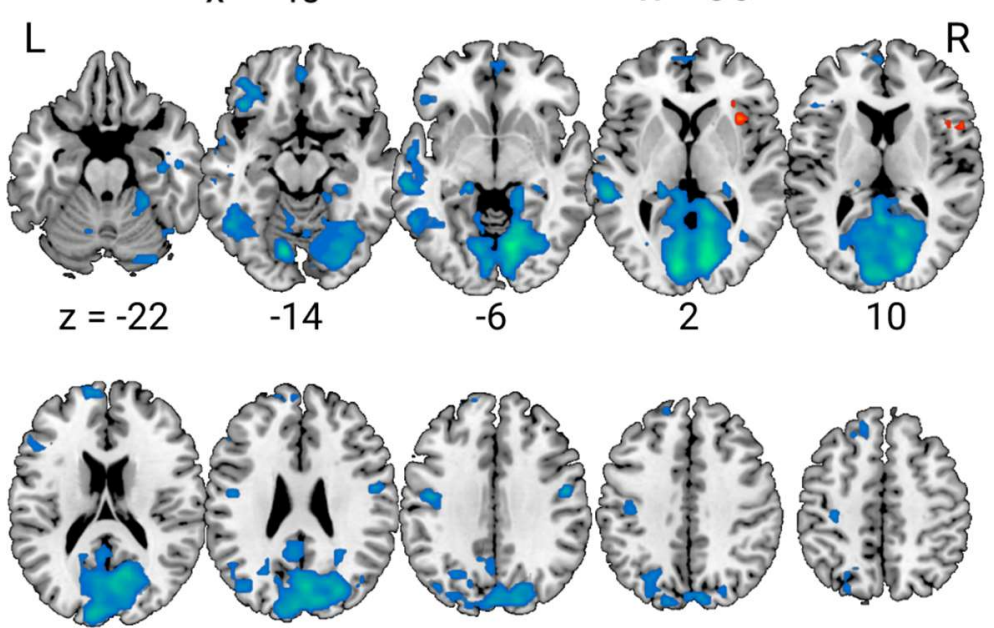

18

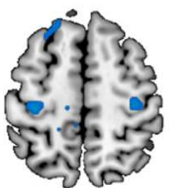

56

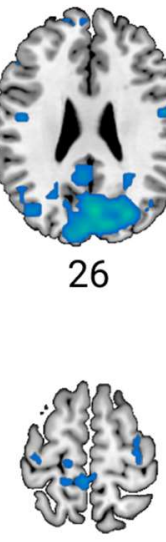

64

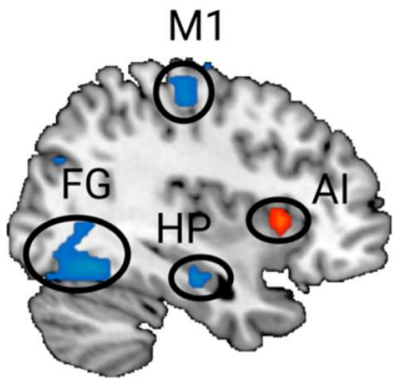

$$
x=36
$$



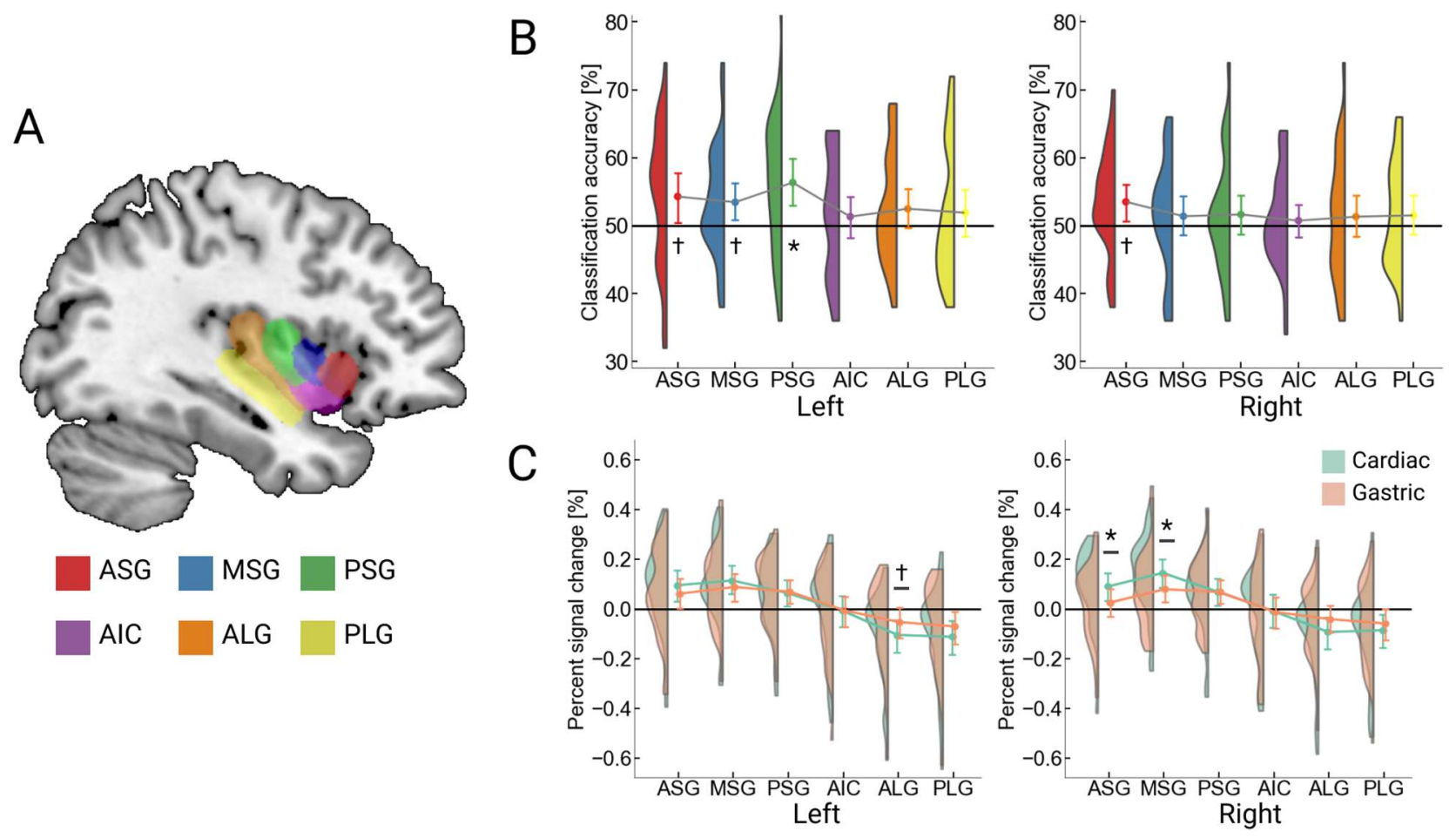